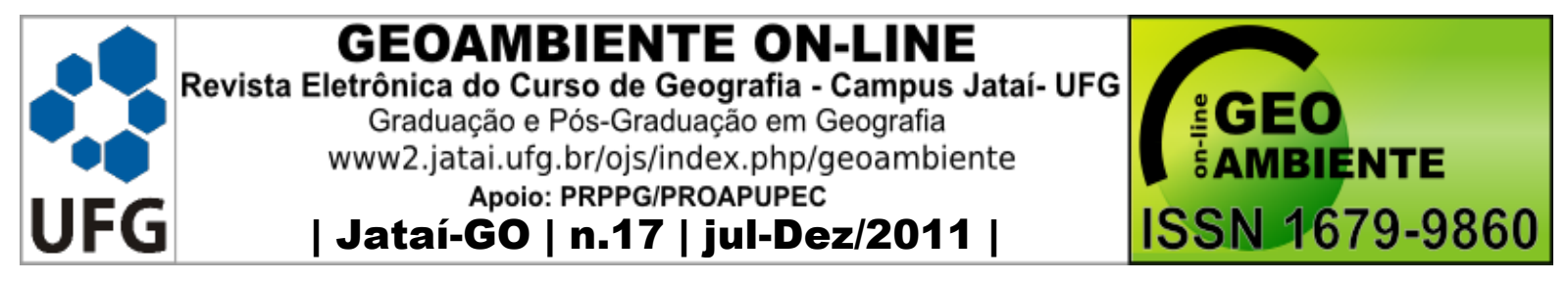

\title{
ANÁLISE DA VIABILIDADE DA OBSERVAÇÃO DE AVES COMO FERRAMENTA PARA EDUCAÇÃO AMBIENTAL E ECOTURISMO NO PARQUE ESTADUAL DA ILHA DO CARDOSO, SÃO PAULO
}

Carolline Zatta Fieker ${ }^{1}$, Matheus Gonçalves Reis ${ }^{1}$, Olivia Tavares Dias ${ }^{2}$, Danilo Janczur Tomaz $^{3}$

(1 - Universidade Federal de São Carlos, Pós-Graduando em Ecologia e Recursos Naturais, Departamento de Ecologia e Biologia Evolutiva - Laboratório de Aves Neotropicais, carolfieker@gmail.com; matheus.reis@ gmail.com, 2 - Universidade Federal do Mato Grosso do Sul, Pós-Graduando em Ecologia e Conservação, oliviadias85@hotmail.com; 3 Universidade Federal de São Carlos, Centro de Ciências Biológicas e da Saúde, danilojtomaz@gmail.com )

\section{Resumo}

Inúmeras estratégias são utilizadas visando mitigar as ameaças à biodiversidade. A criação de unidades de conservação para preservação ambiental deveria ser concomitante à implantação destas estratégias, assim como em localidades onde o meio urbano encontra-se próximo a áreas de alta relevância ecológica. O presente trabalho coletou dados ecológicos sobre a avifauna da região de Cananéia, São Paulo, inserida no contexto dos ambientes costeiros estuarinos. O objetivo é oferecer análises sobre a observação de aves, as quais possam subsidiar a elaboração de planos de visitação em dois âmbitos: educação ambiental regional e ecoturismo como fonte de renda alternativa. O total de 129 aves amostradas em uma semana de inverno, em diferentes ambientes, indica alta atratividade regional para observadores de aves. Cinco ambientes contribuíram com a diversidade de maneiras distintas. Tais diferenças são evidenciadas tanto no tempo gasto para obtenção de listas de espécies, quanto nas singularidades das comunidades. Ambientes urbanos podem ser bem aproveitados em vista da facilidade espaço-temporal de registro de espécies, além da riqueza considerável, enquanto ambientes marinhos são mais atrativos devido às diferenças na comunidade observada. A restinga possui elementos presentes em outros ambientes, o que permite tempos curtos de

Artigo recebido para publicação em 10 de Fevereiro de 2011

Artigo aprovado para publicação em de 20 Dezembro de 2011 


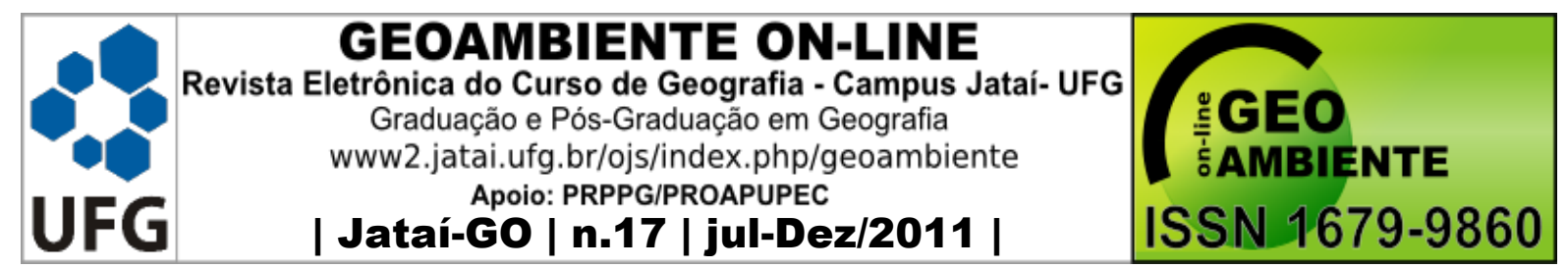

permanência, ao passo que floresta e mangue, por exemplo, exigiriam maior investimento durante visitas. O contexto regional pode ser considerado ideal para ambas as atividades, educação ambiental e ecoturismo, reservando a cada uma as considerações específicas quanto ao aproveitamento de determinados elementos ecológicos.

Palavras-chave: avifauna, ambientes costeiros, birdwatching, Cananéia.

\section{Abstract \\ ANALYSIS OF BIRDWATCHING FEASIBILITY AS A TOOL OF ENVIRONMENTAL EDUCATION AND ECOTOURISM IN "PARQUE ESTADUAL DA ILHA DO CARDOSO - BRAZIL}

Several strategies can be used to mitigate threats to biodiversity. The implementation of protected areas for environmental conservation should be concurrent with the implementation of those strategies, as well as in localities where the urban environment is close to areas of ecological relevance. This study collected data on the avifauna of Cananéia region, São Paulo state, inserted within the context of estuarine environments. We aim to offer analysis about the observation of birds, which can support the development of visitation plans in two aspects: environmental education at regional context and ecotourism as alternative source of income. A total of 129 birds sampled in a week of winter in different habitats indicates high regional attractiveness for birdwatchers. Five environments had contributed to diversity in different ways. Such differences were evident both in the time it takes to get 10 species recorded in lists, and the singularities in the communities. Urban environments can be well used due to its space-time facility of get species record, in addition to its considerable richness, while marine environments are more attractive due to the differences observed in the community. The restinga vegetation features elements present in other environments, which allows short stay, while Atlantic Forest and Mangroves, for example, would require greater investment during visits. The regional context can be considered ideal for both activities, environmental education and ecotourism, allowing specific consideration about the use of certain ecological elements in each of them.

Keywords: avifauna, coastal environments, birdwatching, municipality of Cananéia. 


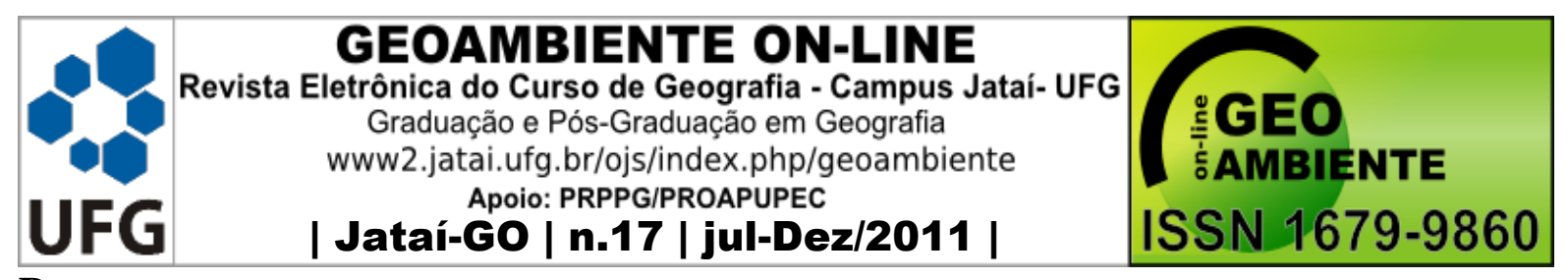

Resumen

\section{ANÁLISIS DE LA VIABILIDAD DE LA OBSERVACIÓN DE AVES COMO UNA HERRAMIENTA PARA LA EDUCACIÓN AMBIENTAL Y ECOTURISMO EN EL “PARQUE ESTATAL DA ILHA DO CARDOSO”, ESTADO DE SÃO PAULO}

Diversas estrategias son utilizadas en la búsqueda para mitigar las amenazas de la biodiversidad. La creación de áreas protegidas para la protección del medio ambiente debe ser concurrente con la aplicación de estas estrategias, así como en los lugares donde la zona urbana es vecina de áreas de gran interés ecológico. En este estudio, fueron obtenidos datos ecológicos sobre la avifauna de la región de Cananéia, estado de São Paulo, en el contexto de los ambientes estuarinos costeros. El objetivo es proporcionar un análisis acerca de la observación de aves que pueda apoyar la preparación de los planes de visita en dos ámbitos: educación ambiental regional y el ecoturismo como una fuente alternativa de ingresos financieros. El total de 129 aves registradas en una semana de invierno, en diferentes ambientes, indica alta atracción regional para los observadores de aves. Cinco ambientes han contribuido de maneras distintas con la diversidad. Estas diferencias son evidentes tanto en el tiempo para obtener las listas de especies, como en la singularidad de las comunidades. Las zonas urbanas pueden ser bien utilizadas en vista de la facilidad espacio-tiempo para registrar aves, además de una riqueza considerable, mientras que los ambientes marinos son más atractivos debido a las diferencias observadas en la comunidad. La restinga cuenta con los elementos presentes en otras áreas, lo que permite estancias de corta duración, mientras que los bosques y pantanos, por ejemplo, requeriría una estancia más larga durante las visitas. El contexto regional puede ser considerado ideal para ambas actividades, la educación ambiental y el ecoturismo, reservando a cada una las consideraciones específicas sobre el uso de ciertos elementos del medio ambiente.

Palabras-clave: avifauna, ambientes costeros, birdwatching, Cananéia.

\section{Introdução}

Uma das tentativas globais para a solução de ameaças à biodiversidade tem sido a criação de áreas de proteção ambiental (Terborgh e Schaik, 2002). No Brasil, de acordo com o Sistema Nacional de Unidades de Conservação (SNUC), instituído pela lei n 9.985 de 2000, 


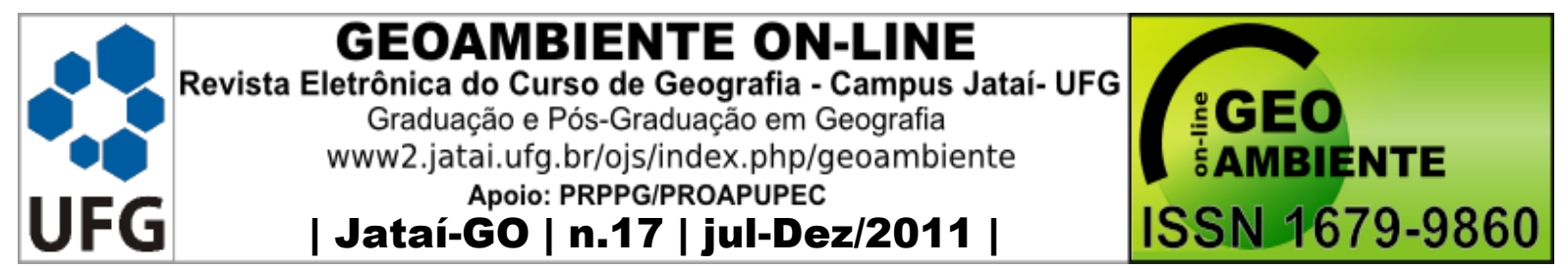

as Unidades de Conservação (UCs) são áreas designadas à preservação e/ou conservação da natureza.

Para que uma área protegida desempenhe seu papel, ao ser criada, geralmente são implantadas em conjunto medidas restritivas em relação ao seu uso, principalmente quanto à exploração de recursos (Wells et al., 1992). Tais medidas podem gerar conflitos, em especial com habitantes das comunidades locais (Primack e Rodrigues, 2001; Wells et al., 1992). Levando em consideração estes problemas e as esferas sociais que afetam, o SNUC (2000) trata de diretrizes que asseguram a participação efetiva das populações locais na criação, implementação e gestão das UCs, além da realização de práticas de Educação Ambiental (EA), e condução das atividades de lazer e do ecoturismo.

Reconhece-se atualmente que, para o sucesso e efetividade de uma UC já implantada, a aprovação e o apoio das comunidades locais são especialmente importantes, uma vez que estas podem se tornar aliadas, cooperando em direção ao alcance do principal objetivo de uma unidade de conservação: a proteção dos recursos naturais, incluindo os bens e serviços ambientais (Nepal e Weber, 1995; Fiallo e Jacobson, 1995). Em casos extremos, mas frequentes, a presença destas comunidades pode se tornar uma ameaça in situ à área de preservação (Primack e Rodrigues 2001; Primack, 2004).

Os comportamentos humanos que afetam a manutenção da diversidade biológica podem ser alterados providenciando-se novas abordagens para a conservação, as quais modifiquem a percepção das pessoas em relação às suas ações, contribuindo com o discernimento sobre qual conduta é a de seu interesse próprio e qual visa o bem comum (McNeely et al., 1995).

Uma atividade econômica que pode funcionar como alternativa de renda às comunidades locais é a prática social do turismo, e também do ecoturismo (Primack, 2004). Contudo, somente quando conduzida de forma adequada, respeitando as características, fragilidades e limites sócio-ambientais da área determinada, e, sobretudo, permeada por ações efetivas de EA, planejamento adequado e participativo, o ecoturismo poderá conduzir a um crescimento sócio-econômico harmonioso e justo da região, à conservação dos recursos naturais e à melhoria da qualidade de viver da população (Tilbury, 1995).

Entre os muitos segmentos do ecoturismo, a observação de aves ou birdwatching se destaca por ser uma atividade de recreação ao ar livre economicamente viável, educacional e 


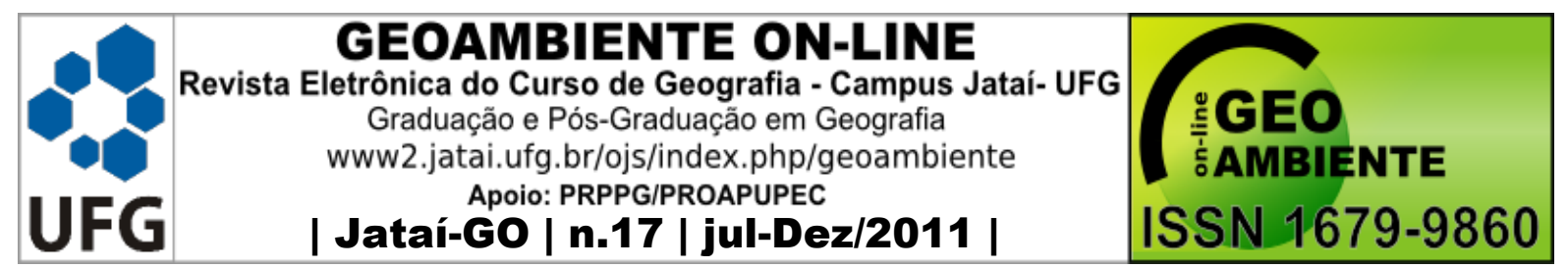

compatível com a preservação ambiental (Farias, 2007). Por ser realizada in situ, em contato direto com a natureza, a observação de aves promove uma gratificante atividade de lazer e descontração, proporcionando aos praticantes recompensas intelectuais, recreativas e científicas (Andrade, 1997).

Turistas e ecoturistas são papéis desempenhados por pessoas que, em visita a áreas naturais, possuem objetivos distintos, assim como suas ações e reflexões direcionadas ao meio natural (Hvenegaard e Dearden, 1998). O ecoturismo, quando comparado ao turismo, resulta em benefícios diretos ou indiretos ao contexto da conservação de áreas naturais (Burger et al., 1995; Hvenegaard e Dearden, 1998). Todo observador de aves pode ser considerado um legítimo ecoturista, que valoriza atividades educativas em que possa aprender algo novo ou que possibilite o desenvolvimento intelectual das populações locais (Farias, 2007), uma vez que estes birdwatchers não buscam esportes ao ar livre nem tampouco perturbam o ambiente com atividades e ações invasivas (Burger et al., 1995). Assim como consideram indispensável a participação da população local nas atividades diretamente ligadas a observação de aves, como, por exemplo, ocupar postos de trabalho criados em função do ecoturismo (e.g. guias de campo), defendem também a distribuição da renda proveniente das atividades econômicas (Scott et al., 1999). Dessa forma, o birdwatching pode estabelecer um desenvolvimento econômico através de trabalhos bem-sucedidos de interpretação da natureza, pois atividades que geram empregos locais criam também outras ocupações de forma indireta como o de artistas gráficos, fabricantes de sinais de identificação, expositores e construtores de centros de visitação (Wearing e Neil, 2001), entre outros.

O presente trabalho tem como objetivo subsidiar a elaboração de medidas futuras que promovam a conservação in situ do Parque Estadual da Ilha do Cardoso, especificamente o uso da observação de aves (Birdwatching) na educação ambiental local e no ecoturismo como fonte de renda alternativa. Por meio da obtenção e análise de dados ecológicos sobre a avifauna regional, sob a perspectiva da distribuição da riqueza e diversidade, oferecemos neste trabalho o suporte logístico fundamental para viabilizar a criação de planos de ecoturismo e educação.

\section{Material e métodos}

Caracterização da área 


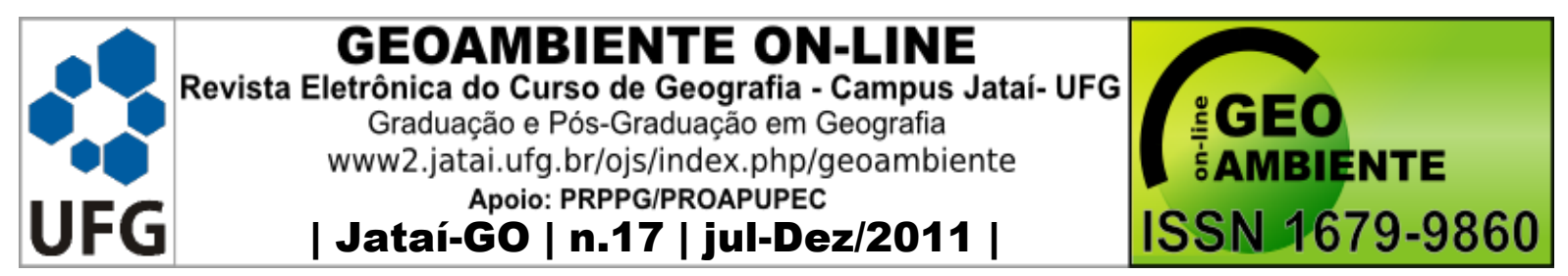

O trabalho foi desenvolvido no município de Cananéia, litoral sul do estado de São Paulo, durante a primeira semana do mês de julho de 2010. Utilizamos áreas ambientalmente desprotegidas e protegidas, principalmente o Parque Estadual da Ilha do Cardoso, criado pelo Decreto $n^{\circ} 40.319$ de 03/07/1962, o qual abrange uma área de 15.100 ha e que tem por base de gestão o SNUC (2000).

A área de estudo está dentro do complexo estuarino lagunar de Iguape-CananéiaParanaguá, o qual se estende pelo litoral desde Peruíbe (SP) até Paranaguá (PR). Visando amostrar os ambientes representativos da região, dentro do contexto ecológico atual dos complexos estuarinos, coletamos dados em cinco ecossistemas distintos.

1. Ecossistemas urbanos: amostramos a zona urbana de Cananéia e área urbanizada da Ilha do Cardoso, onde reside parte da comunidade local (caiçaras) da Unidade de Conservação.

2. Ambientes marinhos: praias de água estuarina (água salgada com forte influência da água doce que chega dos rios continentais), praias próximas a costões voltados para mar aberto, ambiente marinho-estuarino (via transectos em embarcação) e bancos de areia.

3. Mangues (Cananéia e Ilha do Cardoso).

4. Restinga: principalmente a restinga baixa, caracterizada por vegetação predominantemente herbáceo-arbustiva, e restinga arbórea, com indivíduos esparsos com tamanho variando de 3 a 7 metros de altura, em média.

5. Floresta: amostramos a floresta ombrófila densa litorânea, uma das categorias que pertencem ao Domínio da Mata Atlântica.

\section{Procedimentos}

A coleta de dados foi realizada por meio do protocolo conhecido por $m$-species list ou 20-species list, descrito, testado e discutido por Poulsen et al. (1997). Tal método, aqui adaptado e chamado "listas de 10-espécies", consiste em listar os encontros individuais com as espécies de aves, sem repetições, em listas de 10 espécies. Ao finalizar uma lista (quando se anota a $10^{\mathrm{a}}$ espécie), uma nova lista se inicia. Cada listagem é considerada uma unidade amostral, a qual nos forneceu para este trabalho as espécies (dados qualitativos), o número de 


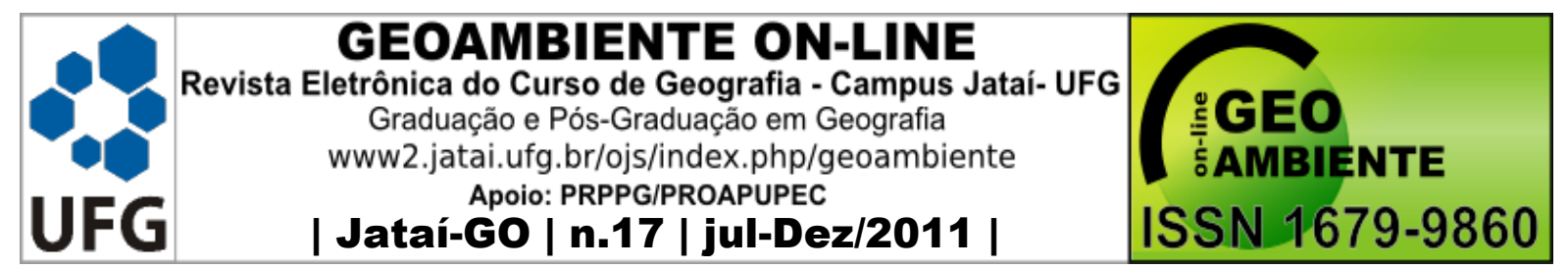

indivíduos observados (encontros auditivos consideramos apenas um indivíduo), o horário do dia, e o tempo em que cada lista demorou a ser preenchida com as 10 espécies. Com cada conjunto de listas obtidas em cada ambiente, calculamos a frequência de ocorrência de cada espécie (número de ocorrência em listas dividido pelo total de unidades amostrais) como maneira de indicar a abundância relativa das aves na região (Santos, 2003). O uso deste tipo de protocolo de amostragem beneficia trabalhos de curto e médio prazo que descrevem comunidades e fornece a proporção com que cada espécie é encontrada (Mackinnon, 1996; Pousen et al., 1997; Fjeldsa, 1999).

As listas de 10-espécies eram obtidas por meio de caminhadas em trilhas, semelhante ao método dos transectos lineares (Bibby et al. 1993, Bibby et al. 1998), sendo que algumas das premissas do método de transectos não eram válidas ou não eram necessárias. Pontos de grande diversidade (hotspots em pequena escala), como um aglomerado de indivíduos arbóreos zoocóricos em frutificação, por exemplo, necessitam de maior atenção e tempo de coleta de dados visando qualidade dos mesmos e a obtenção do maior número possível de registros (Parker e Carr, 1998). Este protocolo de amostragem permite, sem maiores prejuízos à fidedignidade dos dados coletados e caracterização do meio natural, que os observadores parem durante curtos intervalos de tempo, ou mesmo que retornem à coleta de dados na mesma área em um outro dia ou horário (Pousen et al., 1997).

Durante sete dias, os cinco tipos de ambientes foram visitados alternadamente em quase todo fotoperíodo diurno. Categorizamos posteriormente as unidades amostrais dentro de três períodos de amostragem definidos: (a) do início da manhã até às 11:20 horas; (b) o horário mais quente do dia, das 11:21 às 14:40 horas; e (c) final da tarde, à partir das 14:41 até o pôr do sol.

Utilizamos como descritores dos ambientes índices que medem a diversidade, específicos para os tipos de dados coletados (Magurran, 2004). Estimamos a riqueza máxima por meio de estimadores quadráticos de riqueza (Santos, 2003; Magurran, 2004). O delineamento amostral permitiu também análises multivariadas entre as áreas e entre as unidades amostrais de cada área (Sutherland et al., 2008), as quais foram feitas com o uso do programa PAST (Hammer et al., 2001) e Bioestat (Ayres et al., 2007).

Visando buscar padrões de diferenciação entre os ambientes, aplicamos o teste de Kruskal-Wallis. Ainda, por meio de comparações pelo método de Dunn (Hammer et al., 


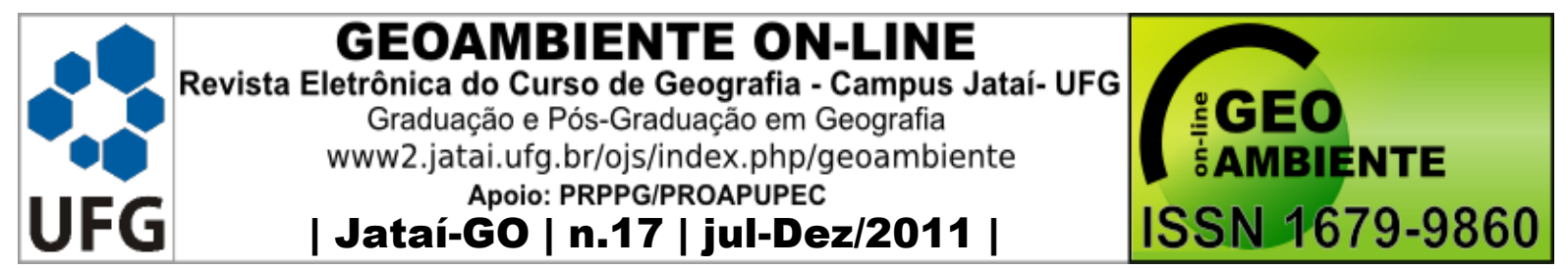

2001), verificamos entre quais ambientes específicos houve diferenças significativas. Para uma análise mais clara da distribuição dos grupos de unidades amostrais, realizamos a análise multivariada conhecida por Detrended Correspondence Analysis (Análise de Correspondência Destendenciada, ou Retificada). Tal método utiliza similaridades nas associações de espécies para ordenar os grupos, e é derivado da Análise de Correspondência, contudo é mais refinado por reduzir a compressão no primeiro eixo e a distorção no segundo e terceiro eixos (Hammer et al., 2001). Ao considerarmos a similaridade entre os ambientes, utilizamos dados da Frequência de Ocorrência (F.O.) de cada espécie em cada local para confeccionar um dendrograma. Utilizamos a Análise de Conglomerados (dentro da análise multivariada de dados) como meio para obtermos o grau de semelhança entre as áreas. O algoritmo escolhido foi a análise pareada ou de grupos pareados, com a medida de similaridade oferecida pelo índice de Morisita, o qual leva em consideração a natureza dos dados de abundância (F.O.).

Todos os dados coletados foram confrontados com Sick (1997), Sigrist (2006) e Stotz et al. (1996), como a distribuição e ecologia de aves neotropicais em ambientes costeiros. Em campo, as espécies foram registradas de acordo com a lista de aves do Brasil elaborada pelo Comitê Brasileiro de Registros Ornitológicos (CBRO, 2009).

\section{Resultados}

Analisamos neste trabalho os dados obtidos em 101 unidades amostrais (listas de 10espécies), realizadas em cinco ambientes distintos. A quantidade de listas em cada ambiente, bem como o tempo médio de obtenção de cada lista, está representada na Tabela 1.

Tabela 1. Unidades amostrais obtidas em cada ambiente estudado e tempo médio de duração para obtenção das listas de 10-espécies (ou tempo médio necessário para o registro de 10 espécies distintas).

\begin{tabular}{lcc}
\hline Ambientes & $\begin{array}{c}\text { Unidades } \\
\text { amostrais }\end{array}$ & $\begin{array}{c}\text { Tempo médio } \\
\text { de obtenção } \\
\text { das listas } \\
\text { (minutos) }\end{array}$ \\
\hline Urbano & 18 & 13.38 \\
Mar & 26 & 24.73 \\
Mangue & 18 & 22.33 \\
Restinga & 16 & 17.43 \\
Floresta & 23 & 23.52 \\
\hline
\end{tabular}




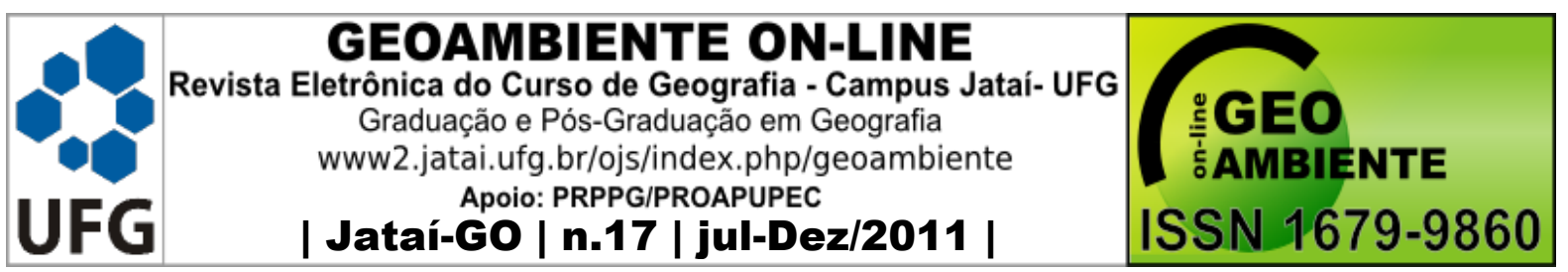

Não houve correlação entre a quantidade de listas obtidas em cada lugar e a média do tempo gasto para se registrar cada grupo de 10 espécies (unidades amostrais). Sendo r $($ Pearson $)=0.73$, e $\mathrm{p}=0.1613$, aceita-se portanto a hipótese de nulidade (não há associação entre os grupos de dados).

Foi obtida uma listagem de espécies que ocorrem na região. Registramos nas 101 unidades amostrais um total de 129 espécies de aves. A riqueza obtida em cada ambiente foi: 55 aves em ambientes urbanos, 25 em ambientes marinhos, 64 no mangue, 46 na restinga e 73 na floresta (Figura 1). Utilizamos quatro estimadores quadráticos de riqueza (ver Tabela 2), sendo que o Jacknife 2, o mais confiável (Santos, 2003; Magurran, 2004), indicou que a riqueza máxima para a região amostrada, levando-se em consideração a época de amostragem (inverno) e as áreas percorridas, é cerca de 145 espécies. O comportamento da coleta de dados foi evidenciado em uma curva de rarefação (Figura 2).

Tabela 2. Estimadores quadráticos de riqueza máxima. Os valores representam o número máximo esperado de espécies para a região (cinco ambientes amostrados) e a época de coleta dos dados (inverno).

\begin{tabular}{llll}
\hline Chao 2: & 135.691 & Chao 2 variance: 0.941176 \\
Jackknife 1: & 144.842 & & \\
Jackknife 2: & 144.928 & \\
Bootstrap: & 138.248 & \\
\hline
\end{tabular}

Figura 1. Riqueza de espécies amostrada em cada ambiente.

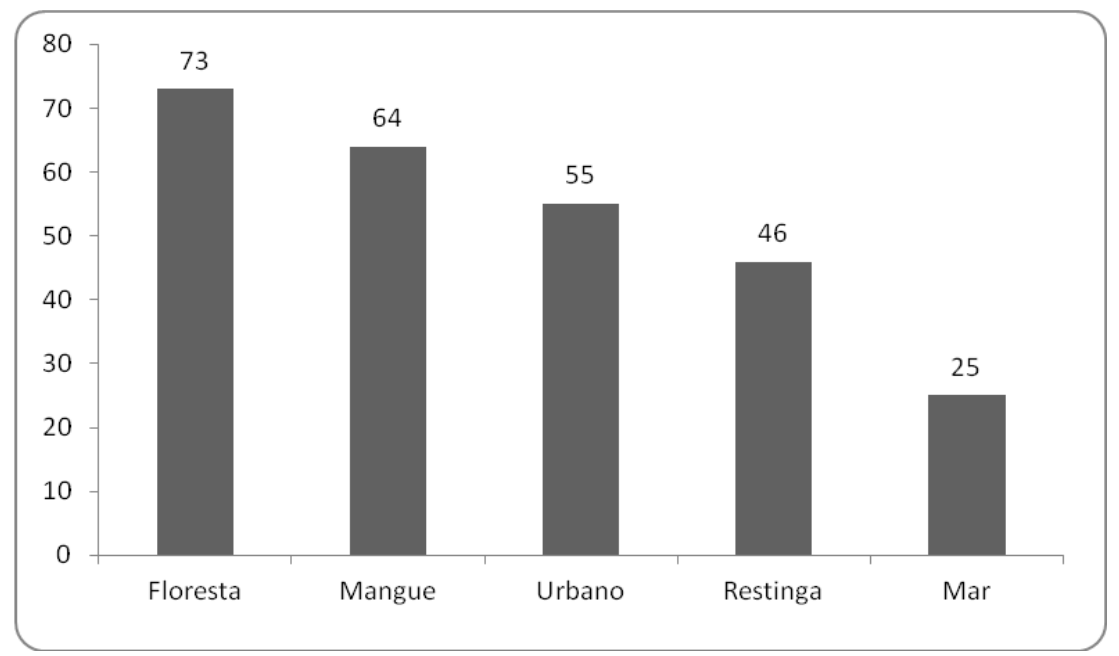




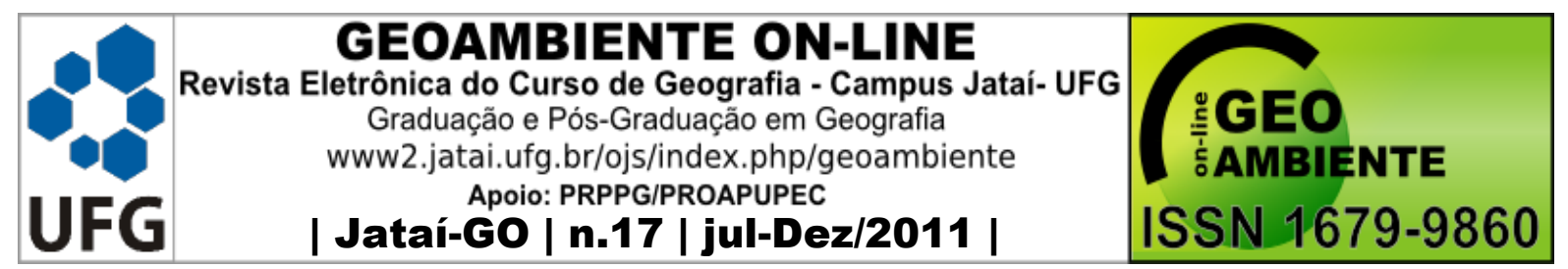

Figura 2. Gráfico do comportamento das amostras quanto à acumulação de novas espécies registradas. A curva de rarefação contém o mínimo e máximo esperados, dentro de um intervalo de 95\% de confiança (com 10 mil reamostragens bootstrap) para os taxa encontrados.

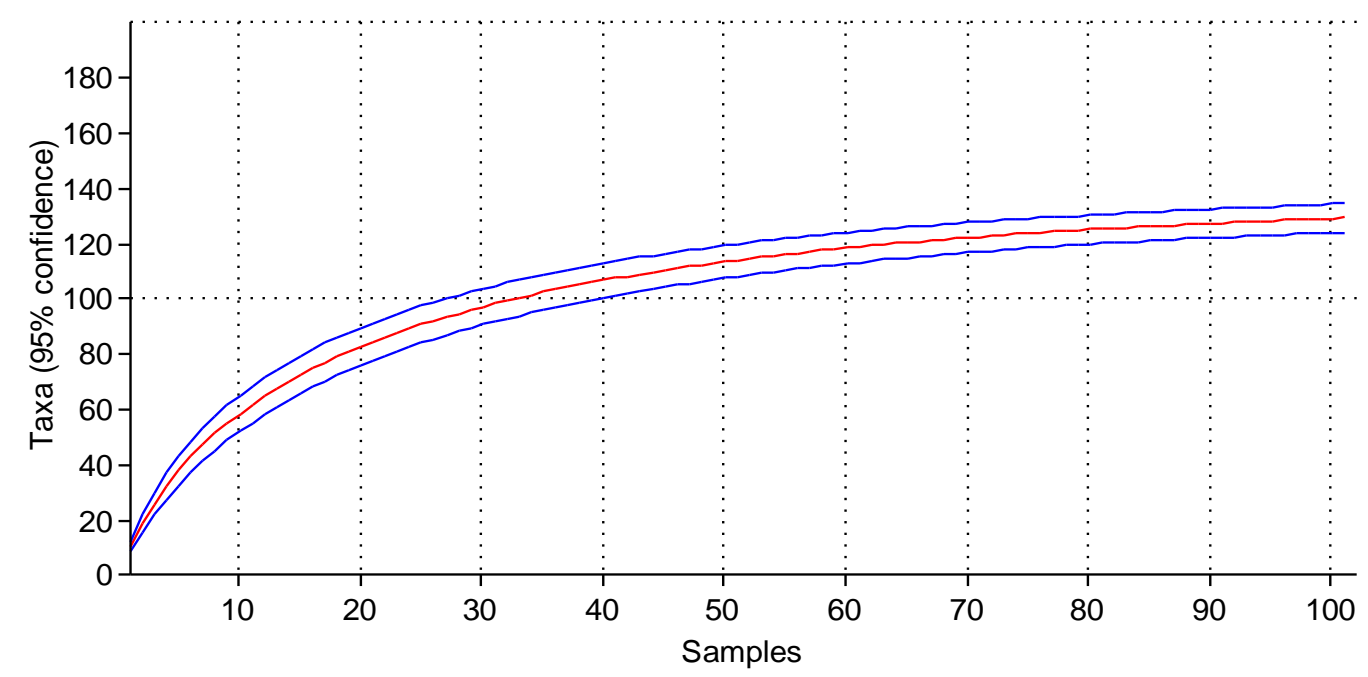

A Tabela 3 traz informações sobre a diversidade amostrada, distribuída em três horários do dia para cada ambiente. Os valores indicados no gráfico (Tabela 3) são advindos de um índice (Índice de diversidade de Shannon, Hammer et al., 2001; Margurran, 2004).

Tabela 3. Índice de diversidade de Shannon. Manhã: até 11:20 horas. Meio do dia: 11:21 até 14:30 horas. Tarde: 14:31 horas até o pôr do sol.

\begin{tabular}{lccccc} 
& Floresta & Mangue & Restinga & Urbano & Mar \\
\hline manhã & 4.395 & 4.128 & 3.921 & 3.682 & 2.865 \\
meio do dia & 4.29 & 4.04 & 3.829 & 3.519 & 3.011 \\
tarde & 4.388 & 4.272 & 3.921 & 3.812 & 3.219 \\
\hline
\end{tabular}

Visando buscar padrões de diferenciação entre os ambientes, os resultados obtidos mostraram diferenças significativas $(\mathrm{H}=33.80, \mathrm{GL}=4$ e $p$-valor de Kruskal-Wallis < 0.0001). Também verificamos entre quais ambientes específicos houve diferenças, sendo que o mar se diferenciou das áreas urbanas, do mangue e da floresta. A restinga se diferenciou da floresta. As demais comparações não apresentaram diferenças significativas (Figura 3). 


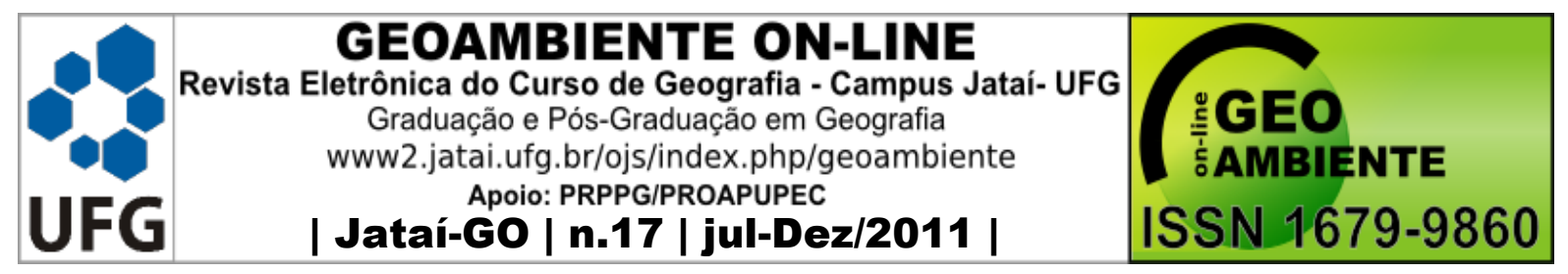

Figura 3. Gráfico do resultado do teste de Kruskal-Wallis, evidenciando diferenças entre alguns ambientes ( $\mathrm{p}<0.05$ ), ou a não existência de diferenças significativas (ns, não significativa). 1. Urbano; 2.Mar; 3.Mangue; 4.Restinga; 5.Floresta. A maior diferença foi encontrada entre 2-5, mar e floresta.

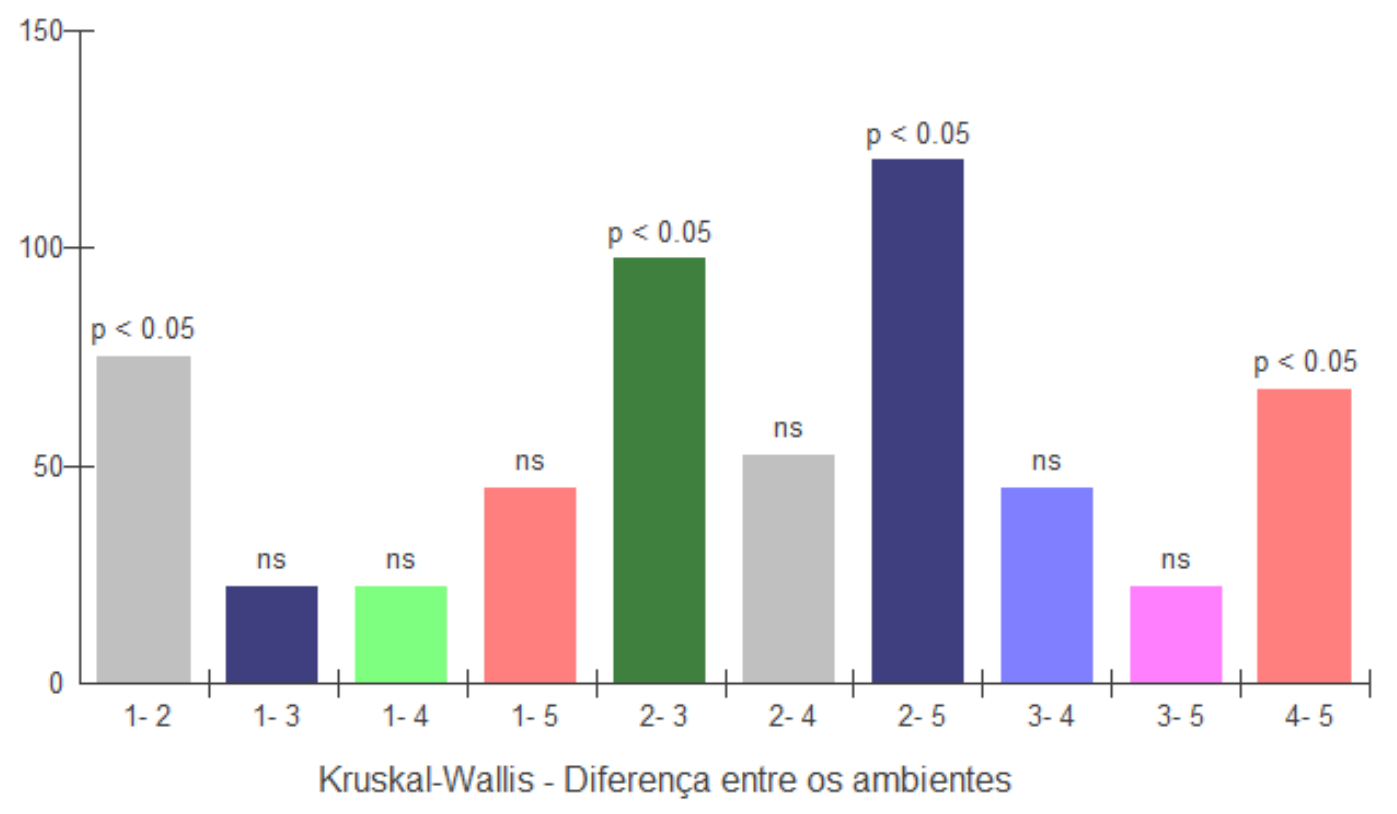

A Figura 4 fornece, graficamente, a distribuição espacial das unidades amostrais dentro de cada grupo. Já a Figura 5 evidencia o grau de semelhança entre as áreas amostradas, sendo que o mangue e a restinga apresentaram a comunidade de aves mais semelhante.

Figura 4. Análise de Correspondência Destendenciada (DCA). Indica a proximidade de cada amostra, e as separa em grupos definidos pelas linhas que margeiam e conectam as amostras limítrofes. 


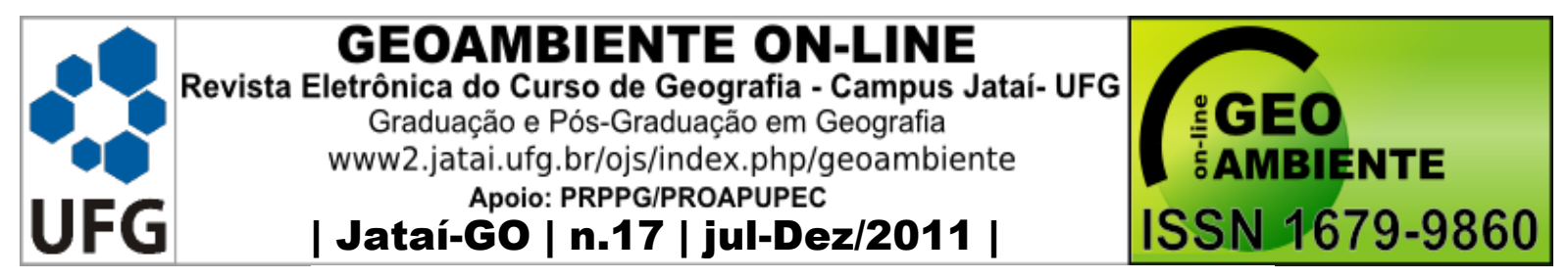

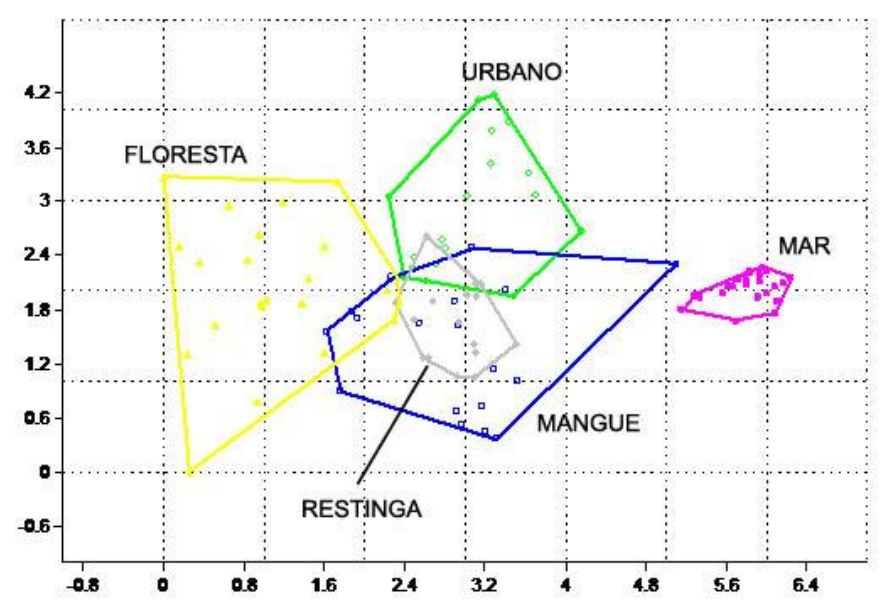

Figura 5. Similaridade entre as áreas: Análise de Conglomerados por pareamento de grupos utilizando o índice de similaridade de Morisita.

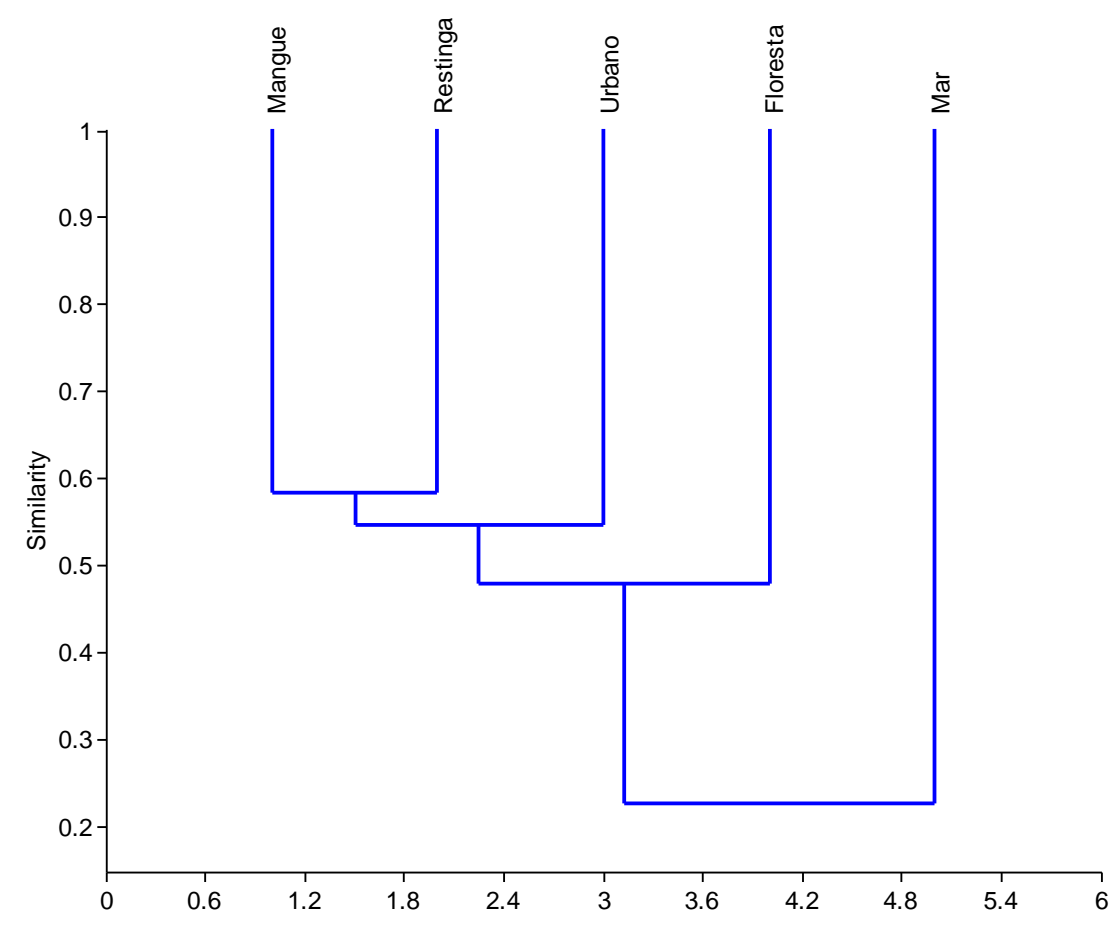

\section{Considerações finais}

O número de espécies observadas em uma semana durante o inverno, em meio a grupos de pessoas, pode ser considerado alto. As 129 espécies contabilizadas somente dentro do protocolo de amostragem das listas de 10-espécies sugerem o grande potencial ecoturístico 


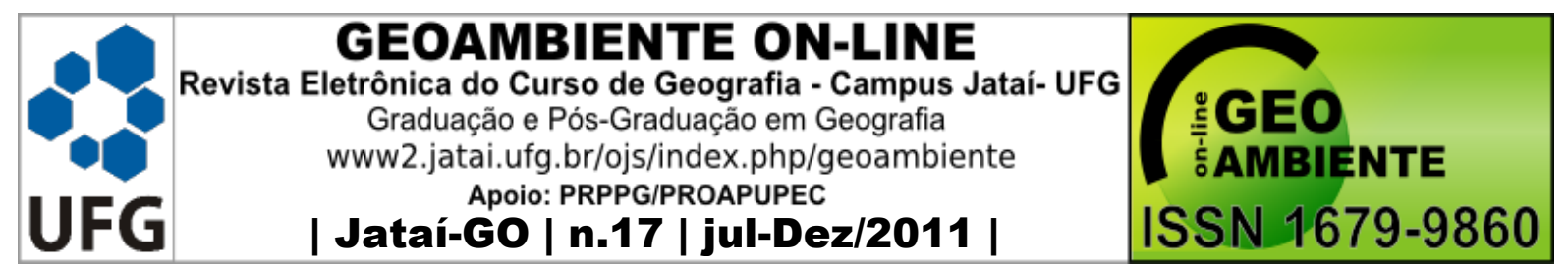

da região. Adicionamos a estes resultados o fato de o Parque Estadual da Ilha do Cardoso ser uma IBA (Important Bird Area) e uma IEA (Important Endemic Area), e fazer parte de um complexo estuarino ainda mais importante do ponto de vista ecológico e da conservação de aves (Wege e Long, 1995; Stotz et al., 1996). Tais informações configuram em direção da atratividade local e regional aos ecoturistas e aos estudantes em geral.

De acordo com a Tabela 1, a cidade é o local onde o tempo para se registrar 10 espécies distintas é menor. As características comportamentais provavelmente explicam este resultado: aves de ambiente urbano possuem um amplo espectro comportamental, podendo utilizar diferentes habitats com a mesma facilidade (Krebs e Davies, 1997; Sick, 1997), além de que estão mais acostumadas (ou condicionadas, Krebs e Davies, 1997) à presença humana constante (Sigrist, 2006). Dessa forma, encontros individuais tornam-se mais constantes e com maior chance de ocorrerem a curtas distâncias, facilitando a detecção mesmo sem equipamentos como binóculos. O mar foi o ambiente que mais necessitou tempo para concluir listas de 10-espécies (Tabela 1), mesmo que a facilidade de identificação das espécies seja maior que a dos demais ambientes. O que explica o tempo médio mais elevado é a baixa riqueza exibida por ambientes marinhos quando comparados aos demais (ver Figura 1). De acordo com o teste de correlação linear de Pearson, a não existência de uma associação estatisticamente significativa entre os dados da Tabela 1 indica que a quantidade distinta de listas obtidas em cada lugar, apesar de diferentes, não foi influenciada pelo tempo de permanência em campo. Assim, guias e educadores podem planejar o tempo de permanência em cada lugar em vista da quantidade de espécies possíveis de serem visualizadas.

A riqueza obtida em cada área pode ser utilizada tanto por um guia quanto por um educador como informação relevante para a escolha de quais localidades visitarem e do tempo de permanência em cada um deles. De acordo com a Figura 1, o fato de a restinga apresentar menos espécies do que o ambiente urbano pode ter sido devido à época de amostragem em adição à dificuldade de detecção de alguns taxa. Quanto aos ambientes marinhos, a baixa riqueza era esperada. Apesar disto, é um ambiente atrativo, pois as aves que o utilizam são de fácil detecção, além dos encontros dependerem quase exclusivamente de contato visual, considerado o tipo mais efetivo de encontro/registro (Sutherland et al., 2008). A permanência de um ecoturista na cidade, ou mesmo a relevância pedagógica de se visitar ecossistemas urbanos, permitem aos interessados aproveitarem o tempo em ambiente urbanizado visualizando a comunidade avifaunística, bem como as possíveis discussões acerca da 


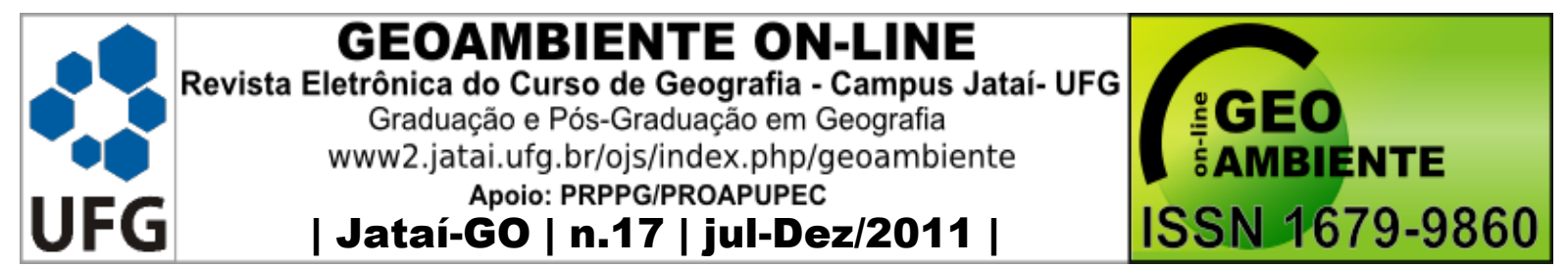

presença de uma quantidade considerável de aves que vivem tão próximas de aglomerados populacionais humanos. Floresta e mangue são áreas muito atrativas, principalmente para Birdwatchers mais especializados, uma vez que a grande riqueza destes lugares pode não ser bem aproveitada por turistas com pouca experiência em identificação de pequenos passeriformes, ou mesmo pela situação de maior dependência das identificações auditivas devido à maior proporção de vocalizações de aves no meio natural em detrimento do contato visual com as mesmas.

As estimativas de riqueza máxima, realizadas por meio de estimadores quadráticos de riqueza, podem nos oferecer interpretações errôneas dos valores obtidos caso não façamos algumas considerações, ou não tenhamos claro o objetivo do uso destas ferramentas estatísticas (Magurran, 2004). A interpretação ecológica para os valores obtidos (Tabela 2) vêm de encontro ao nosso objetivo ao utilizar tais dados: verificar não a riqueza máxima da região, mas sim estimar a quantidade aproximada de aves que, de acordo com nosso método de coleta de dados e o comportamento dos mesmos (ver Figura 2), nós poderíamos ter observado dentro do tempo utilizado (nosso esforço amostral) e exatamente nos locais amostrados. Ou seja, são valores sugestivos, dependentes do espaço-tempo de amostragem, bem como dos procedimentos adotados. Assim, acreditamos que este trabalho poderia ter encontrado até 145 espécies (Jackknife 2, Tabela 2) sob as mesmas condições experimentadas em campo. A riqueza observada, 129 aves, está próxima deste valor. O comportamento da riqueza observada ao longo das 101 unidades amostrais (Figura 2) pode ser utilizada para subsidiar decisões relacionadas ao tempo de permanência na região, ou mesmo o número de espécies que um observador espera ver de acordo com o tempo que ele pode dedicar a isto. Tal análise torna-se ainda mais relevante quando se trata de visitas de instituições de ensino, as quais costumam ter limites mais rígidos de permanência nas áreas.

A Tabela 3 é importante na medida em que revela um padrão bem conhecido da distribuição da atividade das aves (Krebs e Davies, 1997; Sutherland et al., 2008). Exceto em ambientes marinhos, verificamos menor diversidade (segundo o índice de diversidade de Shannon, Hammer et al., 2001; Magurran, 2004) em horários intermediários do dia, geralmente mais quentes e secos se comparados com o início da manhã ou o final da tarde (Sigrist, 2006). Assim, o guia poderá utilizar estes horários com menor diversidade para outras atividades. O mar, onde tal padrão não foi observado, poderia ser visitado nestes horários sem nenhuma perda do potencial de informações possíveis de serem obtidas em 


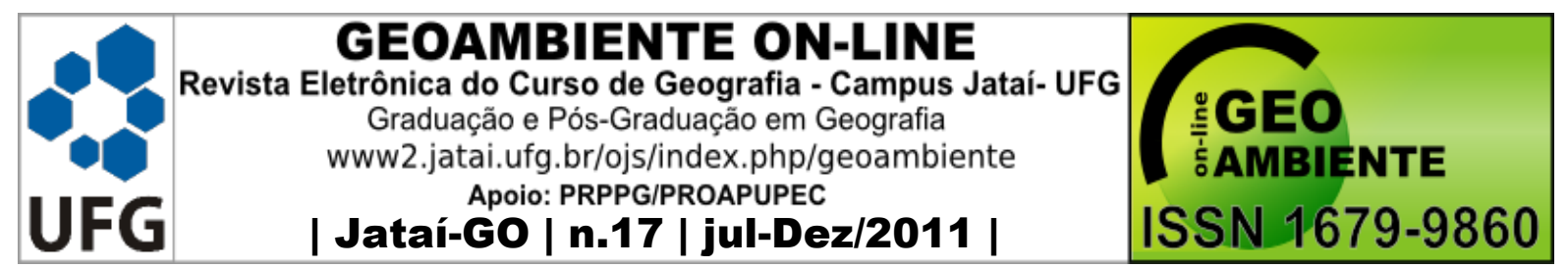

campo. Caso alguma instituição de ensino ou grupo de ecoturistas tenha disponível um número determinado e não alterável de períodos do dia para permanecer na região, os mesmos podem utilizar os dados da Tabela 3 para subsidiar decisões de lugar e período do dia para se dedicarem ao campo e à observação da natureza.

O teste de Kruskal-Wallis indicou que alguns ambientes amostrados são diferentes de outros (ver Figura 3). Tais diferenças significativas nos oferecem mais questões relacionadas ao planejamento de visitas. Surge, pois, a dúvida de quão distintas são as áreas, ou em que direção e sentido ocorrem tais diferenças, e até diferenças dentro de cada área ou similaridade entre todas. Possíveis respostas nos orientam a não super-amostrar determinados ambientes já que alguns destes possuem alta similaridade com outros.

Em vista disto, recorremos à Análise de Correspondência Destendenciada (Figura 4), a qual forneceu um gráfico no qual cada amostra (lista de 10-espécies) é um ponto disperso no espaço. Pode-se visualizar, portanto, agrupamentos (ambientes) e a dispersão das amostras dentro de cada um. Observamos que as amostras correspondentes aos ambientes marinhos são as mais diferenciadas das demais. Assim, mesmo que o mar possua menor riqueza (Figura 1), as amostras obtidas neste ambiente o diferem dos demais significativamente (Figuras 3 e 4), sendo indispensável a visita a ecossistemas marinhos. A floresta é um ambiente altamente atrativo, que contém tanto elementos únicos quando características amostrais semelhantes a outros ambientes.

O mesmo pode ser dito para as localidades com aglomerados humanos. O mangue se assemelha aos ambientes anteriormente citados, ao mesmo tempo em que possui amostras distantes dos mesmos, bem como algumas que se aproximam do mar. De todos os ambientes, pode ser considerado o mais abrangente e cujas amostras podem ser mais dispersas, mesmo aproximando-se de outros grupos. Já a restinga, todas as suas amostras poderiam também ser obtidas visitando-se outros ambientes (exceto o mar), e principalmente o mangue. Assim, no caso de se planejar visitas, o tempo destinado à restinga poderia ser menor, sem grandes prejuízos. Por ser considerado um ecossistema ecologicamente importante, pouco estudado e dentro de um contexto estruturalmente distinto (Stotz et al., 1996), a restinga poderia ser preservada, evitando percorrer muitas trilhas dentro dela, permanecendo assim menos tempo em campo. Outra análise multivariada realizada, agora com o intuito de verificar a similaridade entre ambientes, foi a Análise de Conglomerados (ver Figura 5). 


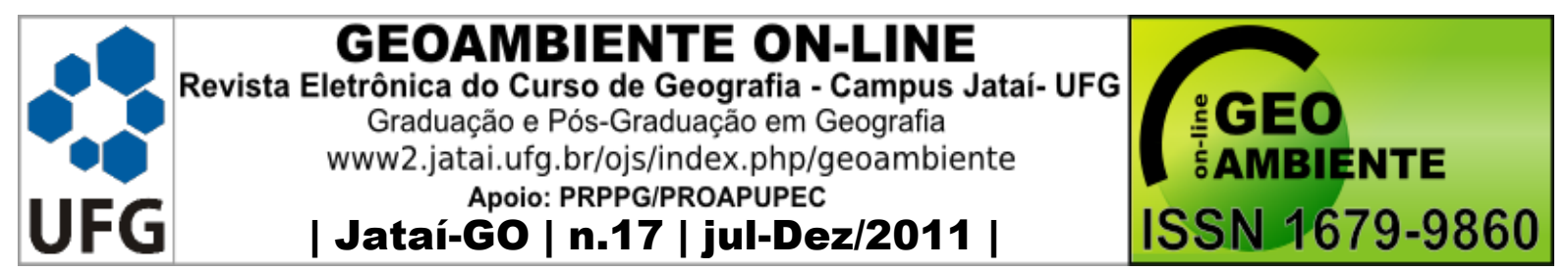

O gráfico obtido corrobora as expectativas e também os resultados anteriores, o que contribui para um julgamento mais robusto por parte de guias e educadores. A restinga poderia ser substituída, por exemplo, pelo mangue e pelo ambiente urbanizado, em termos de similaridade entre os mesmos. Ou, como citado anteriormente, o tempo dedicado a ela ou a área percorrida poderia ser menor. Entretanto, considerações ecológicas durante visitas sobre a singularidade deste ambiente poderiam ser prejudicadas, ou ignoradas, comprometendo a compreensão do todo ecossistêmico regional (Hvenegaard e Dearden, 1998). Novamente o ambiente marinho se mostra diferenciado dos demais, apresentando baixa similaridade (Figura 5).

Sumarizando, os dados oferecidos pelo presente trabalho podem ser utilizados como norteadores de planos de ecoturismo e educação ambiental. A análise de cada tabela ou gráfico subsidia a tomada de decisão durante o planejamento de visitas, tanto com a finalidade de educação ambiental somente, ou o ecoturismo como fonte de renda alternativa na região. Uma vez que ambas as atividades, as quais podem ser aplicadas em conjunto, são estratégias de conservação efetivas e em concordância plena com os objetivos de determinadas unidades de conservação, esperamos contribuir em última instância com a conservação ambiental da região de Cananéia, em especial do Parque Estadual da Ilha do Cardoso.

\section{Agradecimentos}

Agradecemos à CAPES, ao CNPq, ao Programa de Pós-Graduação em Ecologia e Recursos Naturais, e ao Instituto Florestal (Secretaria do Meio Ambiente do Estado de São Paulo), pela concessão dos recursos e infra-estrutura necessários para o desenvolvimento das práticas didáticas no Parque Estadual da Ilha do Cardoso. Agradecemos também o apoio de Thiago Borges Conforti, gestor da Unidade de Conservação "Parque Estadual da Ilha do Cardoso". Somos gratos aos revisores deste artigo pelas valiosas correções, e à amiga Magda Lorena Chilito Amaya pela ajuda na revisão.

\section{Referências}

ANDRADE, MA. 1997. Aves silvestres de Minas Gerais. Littera Maciel, Belo Horizonte. 176pp. 


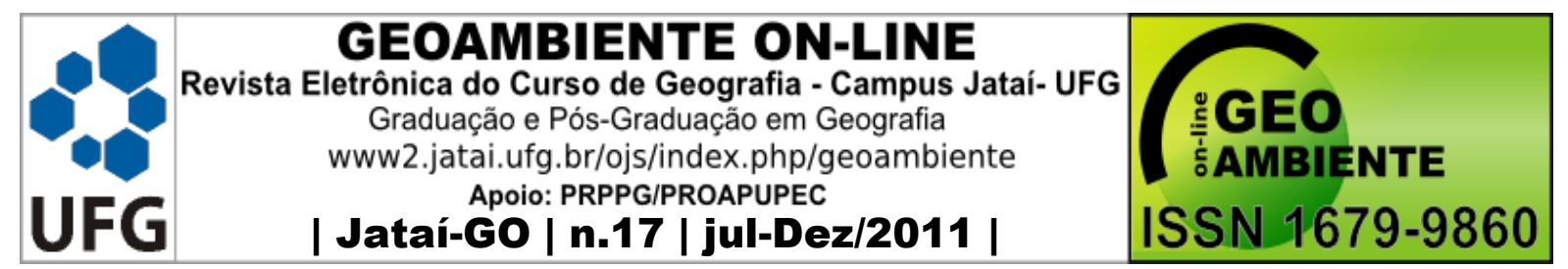

AYRES, M., AYRES Jr., M., AYRES, DL. e dos SANTOS, AS. 2007. BioEstat 5.0: aplicações estatísticas nas áreas das ciências bio-médicas. Sociedade Civil de Mamirauá, CNPq.

BIBBY, CJ.; BURGESS, ND. e HILL, DA. 1993. Bird Census Techniques. Academic Press, London.

BIBBY, CJ.; JONES, M. e MARSDEN, S. 1998. Expedition Field Techniques. Bird Surveys. Expedition Advisory Centre, London.

BURGER, J., GOCHFELD, M. e NILES, LJ. 1995. Ecotourism and birds in Coastal New Jersey: contrasting responses of birds, tourists, and managers. Environmental Conservation. v.22, n.1, p56.

CBRO (Comitê Brasileiro de Registros Ornitológicos). 2009. Listas das aves do Brasil. $8^{a}$ Edição. Disponível em <http://www.cbro.org.br>. Acesso em: [12/05/2010].

FARIAS, GB. 2007. A observação de aves como possibilidade ecoturística. Revista Brasileira de Ornitologia. v.15, n.3, p.474-477.

FIALLO, EA. e JACOBSON, SK. 1995. Local Communities and Protected Areas: Attitudes of Rural Residents Towards Conservation and Machalilla National Park, Ecuador. Environmental Conservation. v.22, n.3, pp 241-249.

FJELDSA, J. 1999. The impact of human forest disturbance on the endemic avifauna of the Undzungwa Mountains, Tanzania. Bird Conservation International 9:47-62.

HAMMER, Ø., HARPER, DAT., e RYAN, PD. 2001. PAST: Paleontological Statistics Software Package for Education and Data Analysis. Palaeontologia Electronica 4(1): 9pp. [on line] Data de acesso: 12/01/2010 Disponível em URL: http://palaeoelectronica.org/2001_1/past/issue1_01.htm.

HVENEGAARD, G.T. e DEARDEN, P. 1998. Ecotourism versus tourism in a Thai National Park. Annals of Tourism Research, v.25, n3, p.700.

KREBS, JR. e DAVIES, NB., 1997. Behavioural ecology. Blackwell Science.

MAGURRAN, AE. 2004. Measuring biological diversity. Oxford: Blackwell Publishing company.

MACKINNON, K. 1996. Managing protect areas in the tropics. Cambridge, UK: IUCN. 


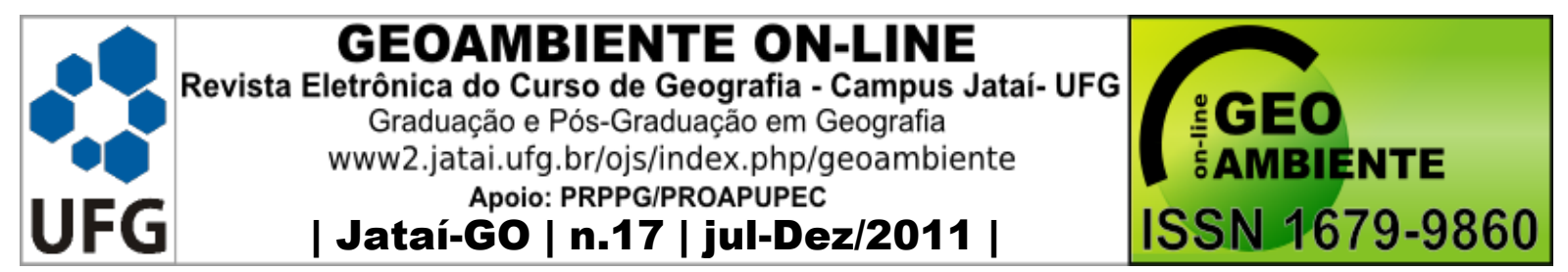

MCNEELY, JA., MILLER, KR., REID, WV., MITTERMEIER, RA. e WERNER, TB. Conserving the world's biological diversity. In WELLS, M., BRANDON, K. and HANNA, L. 1995. People and Parks: Linking Protected Area Management with Local Communities, World Bank WWF / USAID, 98 p.

NEPAL, SK. e WEBER, KE. 1995. Prospects of Coexistence: Wildlife and Local People. Ambio, v.24, n.4.

PARKER, TA.III e CARR, JL. (eds.) 1998. Status of forest remmants in the Cordillera de la Costa and adjacent areas of southwestern Ecuador. Conservation International, RAP Working Paper 2.

POULSEN, BO.; KRABBE, N.; FROLANDER, A.; HIOJOSA, MB. e QUIROGA, CO. 1997. A rapid assessment of Bolivian and Ecuatorian montane avifaunas using 20-species lists: efficiency, biases and data gathered. Bird Conservation International. v.7, n.1 p53.

PRIMACK, RB. 2004. A primer of Conservation Biology. $3^{\text {rd }}$ Edition. Sunderland: Sinauer.

PRIMACK, RB. e RODRIGUES, SE. 2001. Biologia da Conservação. Londrina: E. Rodrigues.

SANTOS, A.J. Estimativas de riqueza em espécies. 2003 In: Cullen Jr., L.; Valladares-Pádua, C.; Rudran, R. (Eds.) 2003. Métodos de estudos em biologia da conservação e manejo da vida silvestre. Editora da UFPR; Fundação O Boticário de Proteção à Natureza.

SCOTT, D., BAKER, SM. and KIM, C. 1999. Motivations and commitments among participants in the Great Texas Birding Classic. Hum. Dim. of Wildlife. v.50, n.4, p.67.

SICK, H. 1997. Ornitologia Brasileira. Edição revista e ampliada por J.F. Pacheco, 2001. Rio de Janeiro: Ed. Nova Fronteira.

SIGRIST, T. 2006. Aves do Brasil: uma visão artística. $2^{\text {a }}$ edição. São Paulo: Editora Avis Brasilis.

SNUC, 2000. - Sistema Nacional de Unidades de Conservação da Natureza: Lei nº 9.985, de 18 de julho de 2000. Brasília: MMA/SBF, 32 p.

STOTZ, DF., FITZPATRICK, JW., PARKER III, TA. and MOSKOVITS, DK., 1996. Neotropical Birds: Ecology and Conservation. University of Chicago Press. 


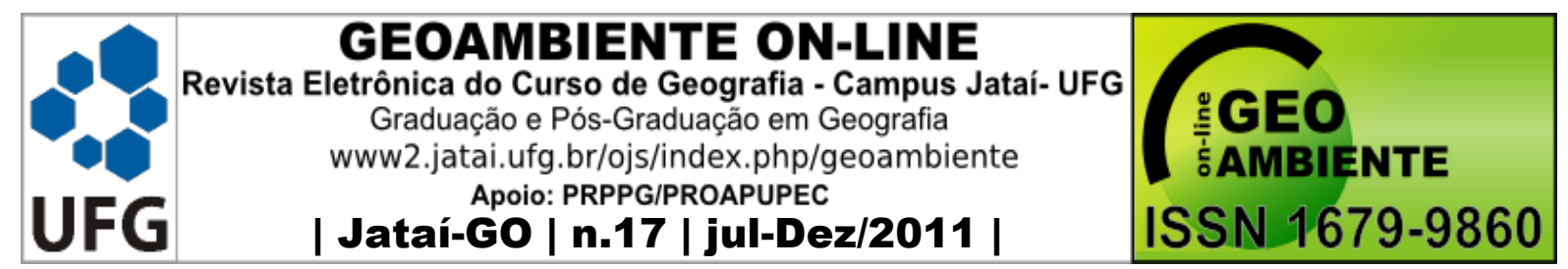

SUTHERLAND, WJ., NEWTON, I., e GREEN, RE. 2008. Bird ecology and conservation: $a$ handbook of techniques. New York: Oxford University Press.

TERBORG. J. e SCHAIK, CV. Porque o mundo necessita de parques. In. TERBORG, J., SCHAIK, CV., DAVENPORT, L. and RAO, M. (ORGS.). 2002. Tornando os parques eficientes: estratégias para a conservação da natureza nos trópicos. Curitiba: Ed da UFPR/ Fundação O Boticário.

TILBURY, D. 1995. Environmental education for sustainability: defining the new focus of environmental education in the 1990s. Environmental Education Research, v.1, n.2, p.195212.

WEARING, S. e NEIL, J. 2001. Ecoturismo: impactos, potencialidades e possibilidades. Barueri, São Paulo: Manole.

WEGE, DC. e LONG, A. 1995. Key areas for threatened birds in the neotropics: BirdLife Conservation Series $n^{\circ} 5$. Washington, DC: Smithsonian Institution Press.

WELlS, M., BRANDON, K. e HANNAH, L. 1992. People and Parks: Linking Protected Area Management with Local Communities, World Bank /WWF / USAID, Washington, DC. 